\title{
Study of Vehicle Handing Stability Based on ADAMS/Car
}

\author{
LI Yuguang $^{1}$, LIANG Changfei ${ }^{1} \&$ WANG Shufen ${ }^{1}$ \\ 1 Mechanical Engineering College Dalian University, Dalian 116622, China
}

Keywords: ADAMS/Car, Handing Stability, Single Lane Change, Simulation

\begin{abstract}
Vehicle handling stability is an important factor which affects vehicle safety. In order to study Vehicle handling stability, we established a dynamic virtual prototype model of a car in ADAMS/Car software, and make simulation of the single lane change on different velocity and steering angle. And we can obtain the vehicle track, yaw rate, lateral acceleration and Side-slip angle curve. This study did a comparative analysis of the various parameters of automotive response character in single lane change, and revealed the vehicle handing stability characteristics. The study provided a theoretical reference for vehicle handing stability control system .
\end{abstract}

\section{Introduction}

The vehicle handling stability is one of the main performances of the car, not only affects the convenience of driving, but also the safety of the car. The study of vehicle handling stability mainly analyze the automobile motion curve of lateral motion, yaw motion and centroid trajectory response,etc[1].

The car is a complicated mechanical system. even simplify the structure and the motion of the vehicle dynamics, the establishment of mathematical model is still very complicate, and the process is also very tedious, which greatly increases the difficulty of the study. In past studies of the vehicle driving stability, many scholars abstracted and simplified the auto structure, and established lots of mathematical models, and made design simulation block diagram to do sinusoidal input simulation in matlab/simulink software, then obtained simulation curves mathematical model of automobile[2, 3]. But in the design of simulation diagram, errors happened because of varied parameters and heavy workload. While establishing the vehicle model in the ADAMS/Car standard interface does not require a complicated mathematical model, intuitive and solid model are enough[4]. We can quickly build and test a vehicle virtual prototype model, which can simulate the actual motion state of automobile, then display important parameters in graphs and obtain the obtain standard test reports in every simulation, which will provide good conditions for the simulation study on vehicle handling stability[5, 6].

\section{Vehicle Simulation Model of ADAMS/Car}

Establishing a vehicle virtual prototype. The front and rear suspension are both independent suspensions. The front suspension is Mcpherson suspension. Engine at the rear, and rear wheel is the driving wheel. Steering system is the Rack and pinion steering structure, which often be used in vehicle. Selecting Magic Formula as tire model.In the vehicle model, taking the parameters of Volkswagen as an example, the vehicle unladen mass assembly is $1450 \mathrm{~kg}$. The wheelbase is $2650 \mathrm{~cm}$, the front tread and the rear tread both are $1530 \mathrm{~cm}$. 


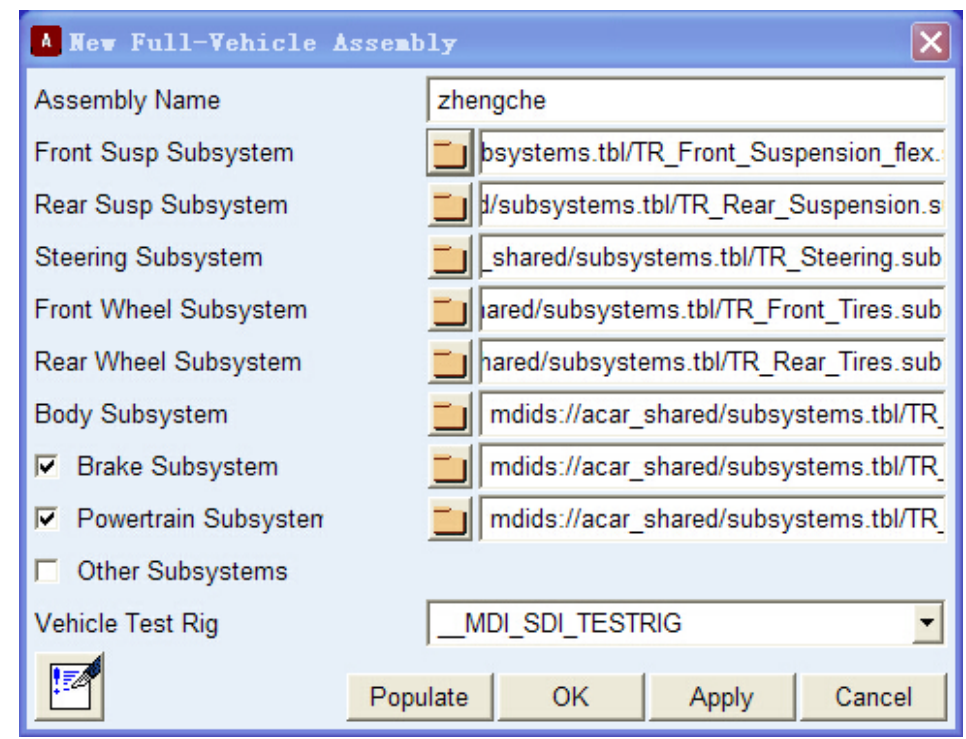

Fig.1 The Process Of Vehicle Assembly

There are vehicle assembly platform. In adams standard interface. The various subsystems import, adams can complete their vehicle assembly[7]. As shown in Figure 1.

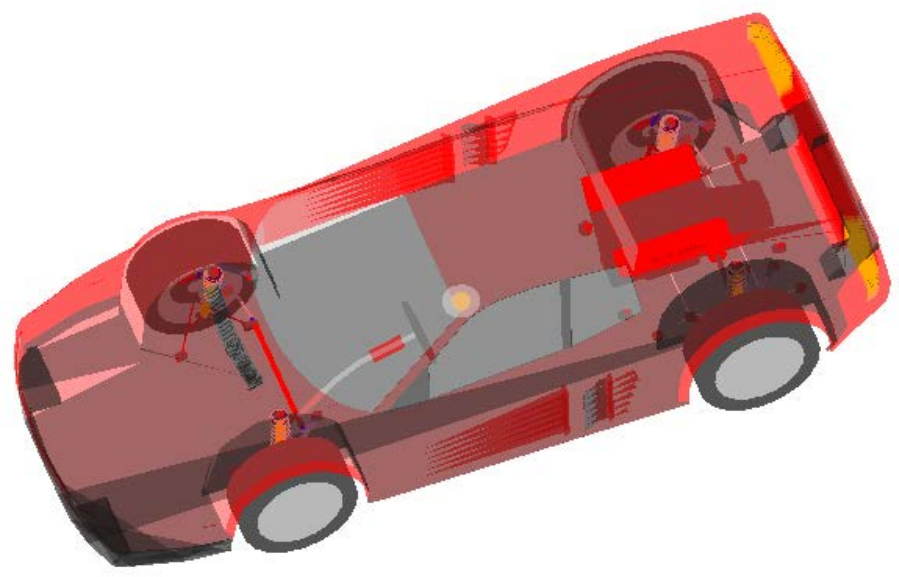

Fig.2 Vehicle Model Assembly

The result is a vehicle model assembly, it can be used to study the dynamic performance of the car.As shown in Figure 2.

\section{Simulation and Analysis of Vehicle Handing Stability}

Vehicle handling stability including handling and cornering stability in traffic lane changing. These two conditions can be achieved by ADAMS/Car vehicle single lane Steering Simulation Test. That is, under the condition of steering wheel angle sinusoidal input, the response of lateral motion, yaw motion and roll motion of the car.

\subsection{Single Lane Change}

Single lane change simulation is a lane-changing action which refers to drive the car through an S curve road within specified time,and simulating lane-changing of the automobile. This kind of simulation is a transient closed-loop response of vehicle overtaking test method. Due to the complication of closed-loop experiment, we usually achieve this by using single cycle sinusoidal angle. We choose low velocity $60 \mathrm{~km} / \mathrm{h}$ and high velocity $120 \mathrm{~km} / \mathrm{h}$,input the steering wheel angle (the maximum wheel angles are different) ,and output curves of driving route,side-slip angle, yaw rate, lateral acceleration and vehicle roll angle [8]. Angular velocity are amplified.

Table.1Fish Hook Driving Simulation Parameters

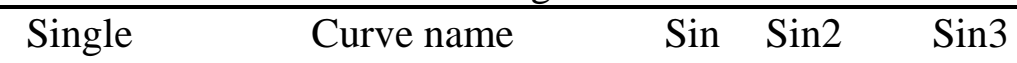




\begin{tabular}{|c|c|c|c|c|c|}
\hline Lane & & 1 & & & \\
\hline Change1 & The steering angle/deg & 60 & 90 & & 0 \\
\hline & Cycle length/ s & \multicolumn{4}{|c|}{2} \\
\hline & $\begin{array}{l}\text { Longitudinal } \\
\text { velocity } / \mathrm{km} / \mathrm{h}\end{array}$ & \multicolumn{4}{|c|}{60} \\
\hline Single & Curve name & Sin & Sine & Sin & Sin \\
\hline Lane & & e1 & 2 & e3 & e4 \\
\hline \multirow[t]{3}{*}{ Change2 } & The steering angle/deg & 60 & 70 & 80 & 90 \\
\hline & Cycle length/s & \multicolumn{4}{|c|}{2} \\
\hline & $\begin{array}{c}\text { Longitudinal } \\
\text { velocity } / \mathrm{km} / \mathrm{h}\end{array}$ & \multicolumn{4}{|c|}{120} \\
\hline
\end{tabular}

\subsection{The Simulation Results and Analysis}

Single lane change simulation 1 is the Simulation of handling stability under low velocity of the car. It obtains the curve of car running track, side-slip angle, yaw rate and lateral acceleration as showed in Fig.3- Fig.6. X-axis is longitudinal displacement, $\mathrm{Y}$-axis is value of response curves which named $\sin 1$, sin2 and $\sin 3$,they are response curves with maximum steering value of 60 degrees, 90 degrees and 100 degrees respectively.

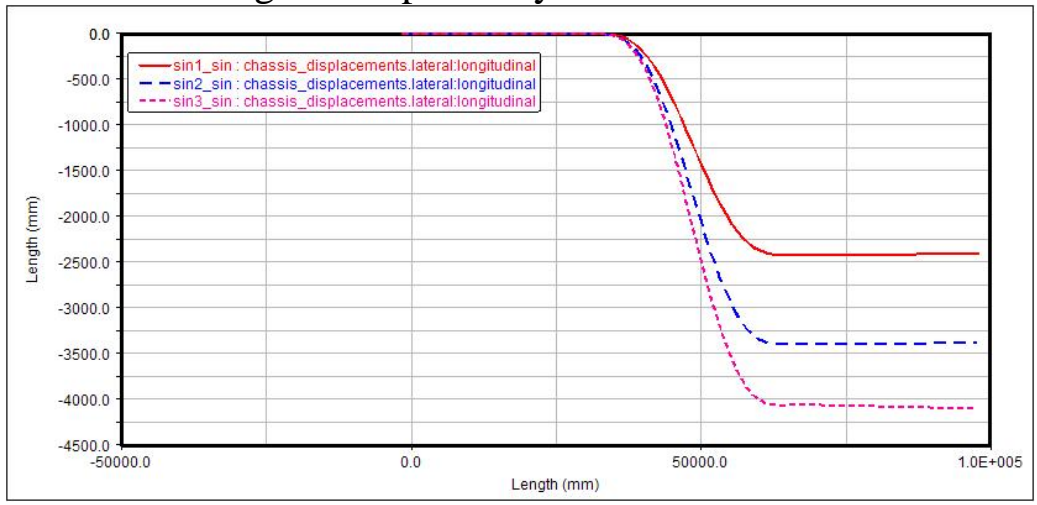

Fig.3 The Centroid Trajectory

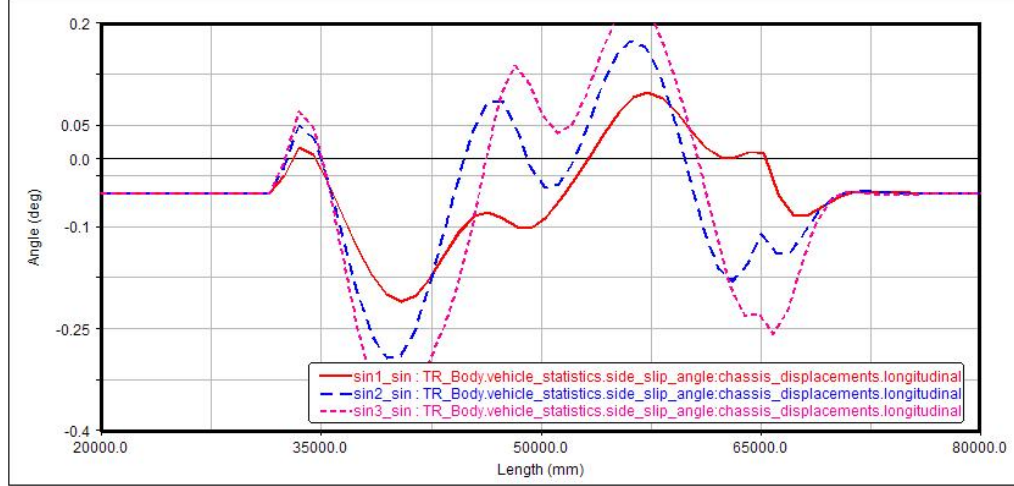

Fig.4 Side-slip Angle Curve 


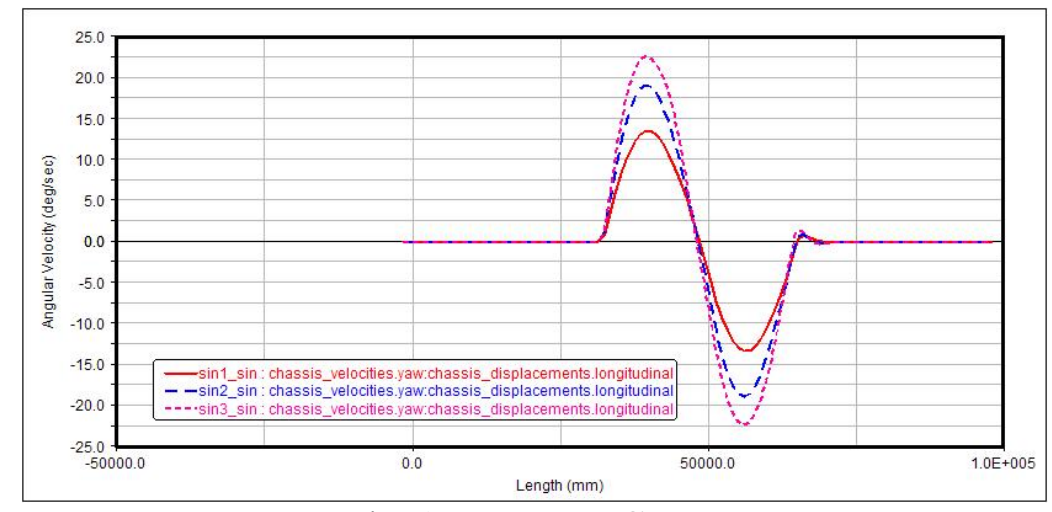

Fig.5 Yaw Rate Curve

In the single lane simulation 1 , figure. 3 shows that when the car driving at low velocity, with the increasing of steering angle, although road-width for vehicle lane changing is increasing, the car still can change the lane accurately. Fig.4-and Fig.6 shows that when the car driving at low velocitys, with the increasing of vehicle steering angle, side-slip angle, yaw rate and lateral acceleration also increasing, which still haven't exceed the range of stability. Therefore, the car can keep good handling and stability at low velocity when turning or changing lanes.

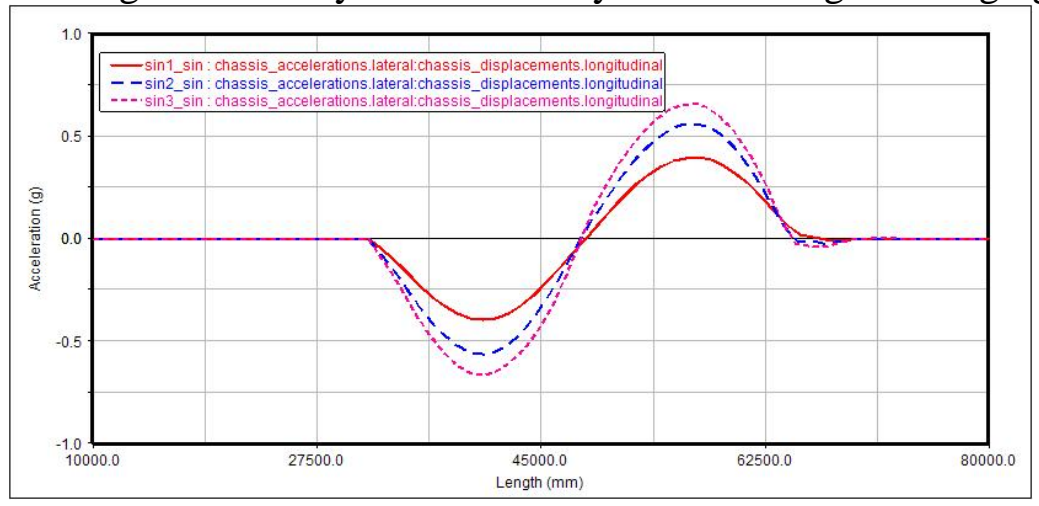

Fig.6 Lateral Acceleration Curve

Single lane change simulation 2 did handling stability simulation of car in high velocity, we can get the car running track, side-slip angle, yaw rate, lateral acceleration and body side angle curve as shown in Fig.7-Fig.10, X-axis is longitudinal displacement in all of Figure, Y-axis is value of response curves which named sine1, sine2, sine3 and sine4, they are corresponding to the maximum steering value 60 degrees, 70 degrees, 80 degrees and 90 degrees respectively.

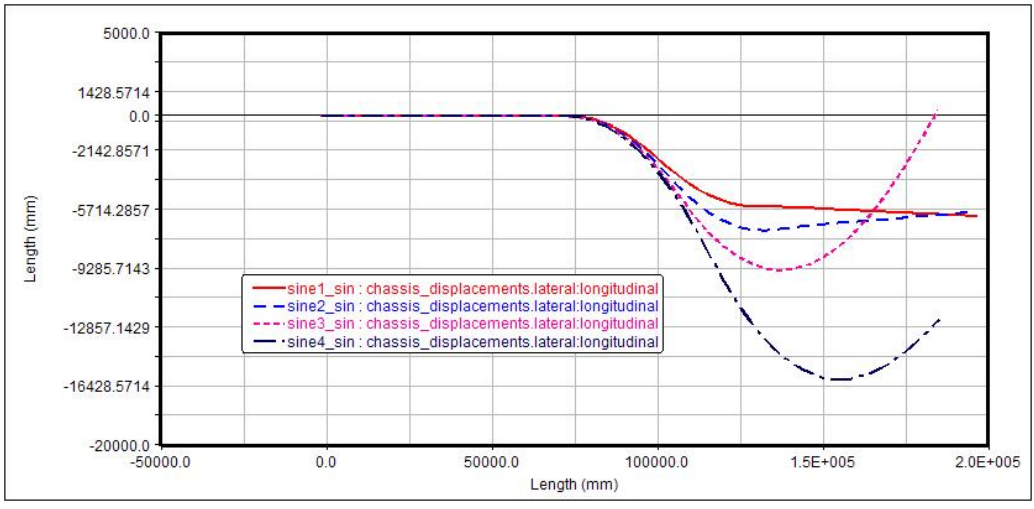

Fig.7 The Centroid Trajectory 


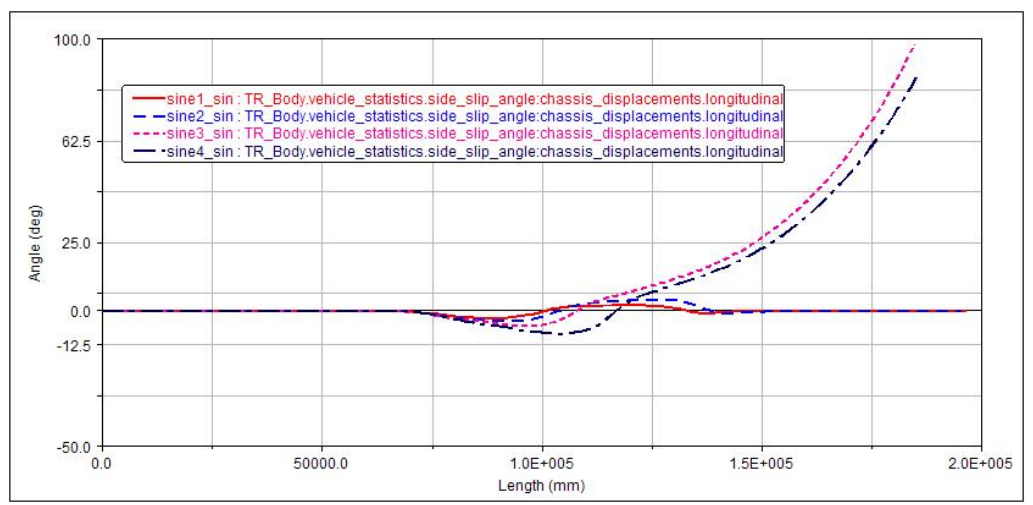

Fig.8 Side-slip Angle Curve

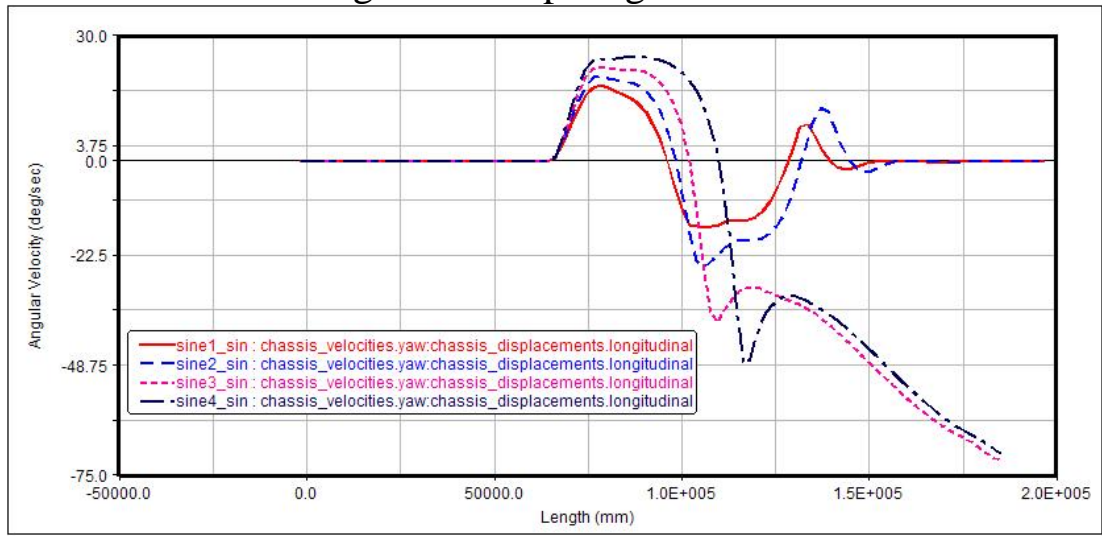

Fig.9 Yaw Rate Curve

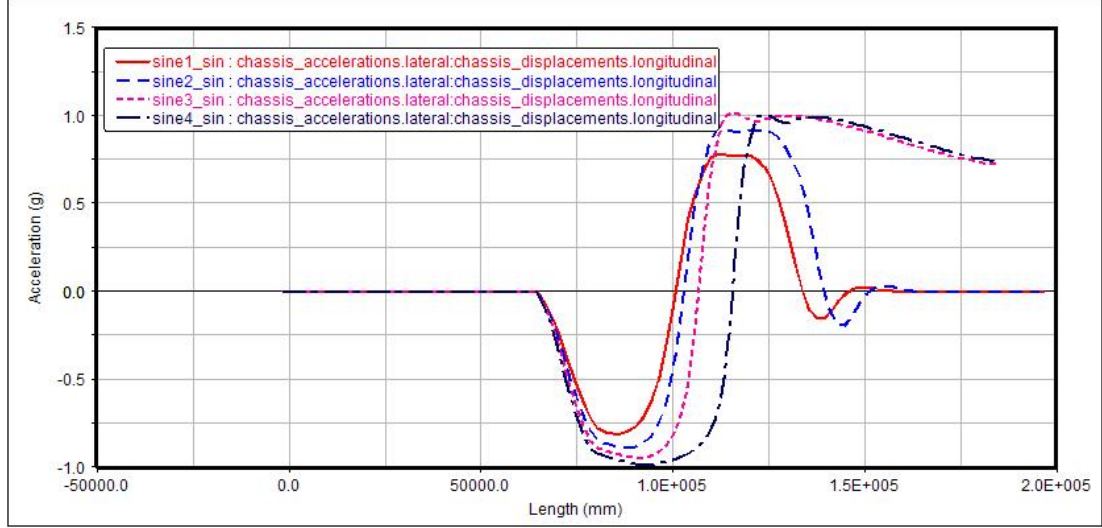

Fig.10 Lateral Acceleration Curve

In the single lane change simulation 2, figure.7 shows that , road-width for vehicle lane changing is increasing, the maximum steering angle of the two cars with 60 degree and 70 degree (sine1, sine2) are still able to complete the lane changing tasks accurately, while the maximum steering angle of two cars are 80 degree and 90 degree (sine3, sine4) achieve the steering angle peak in the range of $130 \mathrm{~m}-150 \mathrm{~m}$, then the car loses its stability, and couldn't running normally. Figure.8-figure.10 shows that when the car doing lane-changing driving at high velocity, with the increasing of steering angle, with the increasing of the automobile steering angle, side-slip angle, yaw rate and lateral acceleration, the two cars (sine1, sine2) still within the stability range. While the other two cars (sine3, sine4), their angle of side-slip and yaw rate of the value increase rapidly in the $120 \mathrm{~m}-130 \mathrm{~m}$, then they lose the stability; the two vehicle lateral acceleration maintain for a long time near the amplitudes, the reducing rate is no longer significant, that is the main reason of lost stability of automobile. Therefore, when car in high velocity turning or changing lanes, too large steering angle may make the car easy to lose the vehicle handling stability .

According to the two single lane simulations, we can find that when car steering and changing lane, reduce the velocity and steering angle can be the safest way, when the car driving at high velocity and doing instant steering lane-changing action, large steering angle intensifies the lateral movement, which bring serious threaten to manipulating security of the 
vehicle. Once beyond the limit condition, performance of the automobile tire and suspension components may not guarantee the handling stability. In this case, you need to add the electronic stability program electronic control system. Reducing lateral angular velocity can be regarded as the main target of control. At the same time, reducing the car velocity and steering angle can be used as an auxiliary[9, 10].

\section{Conclusions}

From the simulation contrast analysis above,we can see that ADAMS/Car as analysis module used specially for vehicle dynamics, because each process from the mechanics modeling to dynamic simulation analysis,are parametric process,it can quickly and easily establish the physical prototype development scheme. Therefore,ADAMS/Car has promising application prospect.Furthermore, according to the above analysis,we can obtain following conclusions .

i) The application of simulation software ADAMS/Car, including the establishment of the vehicle steering system, front and rear suspension assembly, vehicle simulation model of body and tires, car, can simulate more real in the steering wheel to the steering characteristic of sinusoidal input conditions, provides the simulation as an effective method for handling and stability analysis of automobile.

ii) The car in low velocity turning lane changing, along with the increase of the steering angle and vehicle side-slip angle and yaw rate and lateral acceleration change amplitude increasing, still did not exceed the range of stability; the car at high velocity turning or changing lanes too large steering angle to make the car lost control stability. Reduce velocity and reduce the steering angle is the most secure scheme;

iii) Velocity and steering angle will make the vehicle lateral motion increases, performance depends not only on the automobile tire and suspension components to ensure the operation stability, you need to add the electronic stability program electronic control system to regulate and enhance vehicle handling and stability.

\section{References}

[1] S. YIM, Y. PARK and K. YI .Design Of Active Suspension And Electronic Stability Program For Rollover Prevention [J].International Journal of Automotive Technology, Vol. 11, No. 2, pp. 147-153 (2010)

[2] Shin Ae Lee, Byung Hak Kwak . A Study on Optimal Yaw Moment Distribution Control Based on Tire Model [J].SAE paper 2007-01-3563, 2007.

[3] YU Zhisheng.The theory of automobile [M].Machinery Industry Press 2012.9:194 196

[4] Ken Koibuehi, Masaki Ymamoto, Yoshiki Fukad, and Shoji Inagaki,Vehiele Stability Control in Limit Comering by aetive Brake. SAE Paper NO.960487

[5] Yoshiki Fukada.SliP Angle Estimation for Vehicle Stability Conirol.Vehiele System Dyanlnies, 1999, 32(4)

[6] Guo Konghui. Vehicle handling dynamics [M]. Changchun: Jilin Science and Technology Press, 1991

[7]WANG Shuwei.LIU Weiyan. Affect ADAMS/Car Elastic Kinematics Analysis Based on The Vehicle [J]. Automotive Engineers 2011 (3)

[8] OU Jian.Vehicle Dynamics Integrated feedback control simulation system stability[J].Tractors And Farm Vehicles,2010,37(3).

[9] WANG Deping . Theoretical studies of vehicle dynamics stability control [J]. Automotive Engineering2000, 22(1): 7 9.

[10] LI Liang. Research status and development of vehicle dynamics stability control system [J]. Journal of agricultural machinery 2006, 37(2): 141 144. 